\title{
Identification of a novel starch synthase III from the picoalgae Ostreococcus tauri
}

Julieta Barchiesi ${ }^{1}$, Nicolás Hedin ${ }^{1}$, Alberto A. Iglesias ${ }^{3}$, Diego F. Gomez-Casati ${ }^{1}$, Miguel A. Ballicora ${ }^{2}$ and María V. Busi ${ }^{1}, *$

1 Centro de Estudios Fotosintéticos y Bioquímicos (CEFOBI-CONICET), Universidad Nacional de Rosario, Suipacha 531, Rosario (2000), Argentina

2 Department of Chemistry and Biochemistry, Loyola University Chicago, 405 Flanner Hall, 1068 W Sheridan Road, Chicago, IL 60660, USA

3 Laboratorio de Enzimología Molecular, Instituto de Agrobiotecnología del Litoral (UNLCONICET) \& FBCB, Santa Fe (3000). Argentina.

*Corresponding author: María V. Busi

E-mail: busi@cefobi-conicet.gov.ar

Telephone number: Tel: +54 3414371955

Address: CEFOBI. Suipacha 531, 2000, Rosario, Argentina.

\section{Abbreviations:}

GS, glycogen synthase; SS, starch synthase; SSIII, starch synthase III;

ADPGlc, ADP-Glucose; SBD, starch-binding domain;

CBM, carbohydrate-binding module; CD, catalytic domain; UDPGlc, UDP-Glucose.

Ostta, Ostreococcus tauri.

Running title: Novel starch synthase III from O. tauri. 


\begin{abstract}
Hydrosoluble glycogen is the major energy storage compound in bacteria, archaea, fungi, and animal cells. In contrast, photosynthetic eukaryotes have evolved to build a highly organized semicrystalline granule of starch. Several enzymes are involved in polysaccharide synthesis, among which glycogen or starch synthase catalyze the elongation of the $\alpha-1,4$-glucan chain. Ostreococcus tauri, accumulates a single starch granule and contains three starch synthase III (SSIII) isoforms, known as OsttaSSIII-A, OsttaSSIII-B and OsttaSSIII-C. After amino acids sequence analysis we found that OsttaSSIII-C lacks starch-binding domains, being $49 \%$ identical to the catalytic region of the SSIII from Arabidopsis thaliana and 32\% identical to the entire Escherichia coli glycogen synthase. The recombinant, highly purified OsttaSSIII-C exhibited preference to use as a primer branched glycans (such as rabbit muscle glycogen and amylopectin), rather than amylose. Also, the enzyme displayed a high affinity toward ADP-glucose. We found a marked conservation of the amino acids located in the catalytic site, and specifically determined the role of residues R270, K275 and E352 by site-directed mutagenesis. Results show that these residues are important for OsttaSSIII-C activity, suggesting a strong similarity between the active site of the $O$. tauri SSIII-C isoform and other bacterial glycogen synthases.
\end{abstract}

Keywords: O. tauri, starch synthase, protein activity. 


\section{Introduction}

The synthesis of bacterial glycogen and plant starch exhibit several common features. Both involve the action of at least three enzymes, ADP-glucose (ADPGlc) pyrophosphorylase (EC 2.7.7.27), glycogen/starch synthase (EC 2.4.1.21), and starch branching enzyme (EC 2.4.1.18). Starch synthase (SS) catalyzes the elongation of $\alpha-1,4$ glucans by the transfer of glucose units from a sugar-nucleotide donor to the non-reducing end of the growing polyglucan chain [1]. Photosynthetic eukaryotes SSs are GT-B-fold glycosyltransferases classified within the GT5 family in the CAZy database [2, 3], being the archaeal and bacterial glycogen synthase (GS) their closest counterparts [4, 5]. They use ADPGlc as the glycosyl donor. The catalytic region of SS, consisting of a conserved synthase catalytic domain (GT5) and a glycosyltransferase 1 domain (GT1), is encompassed by nearly the entire sequence of the $\sim 60 \mathrm{kDa}$ protein of bacterial GSs, but it corresponds only to the C-terminal portion of SSs from photosynthetic eukaryotes [2]. The $\mathrm{N}$-terminal region of plant and algae SSs has a variable length as well as differences in amino acids sequence among the different isoenzymes. Such differences could be related to the specific functions attributed to each SS isoform.

Three SS subfamilies were reported in O. tauri consisting of one SSI, one SSII and three SSIIIs isoforms; all of them remaining uncharacterized to date. The conservation throughout the evolution of the three SSIII isoforms and the absence of an SSIV isoform could be related to the presence of a single starch granule in this alga, which has similar composition that those starch granules found in higher plants but with a particular partitioning and propagation mechanism $[6,7]$. In plants, the N-terminal region of the SSIII isoform contains three in tandem starch binding domains (SBDs), belonging to the carbohydrate binding module 53 (CBM53) family [3, 8]. These SBDs are non-catalytic modules that present starch binding ability and are involved in modulating the catalytic properties of the enzyme [8-13].

We have previously shown that SSIII isoforms from algae present a variable number of SBDs in their N-terminal region [6]. The model green algae Chlamydomonas reinhardtii for example, presents two SSIII isoforms, containing two and three SBDs respectively. Interestingly, the $O$. tauri SSIII-C isoform sequence stands for an atypical 
SSIII, as it contains the GT5 and GT1 domains but lacks of a SBD polypeptide. In this work we report on the molecular cloning and expression of the OsttaSSIII-C gene, and the production in a highly purified form and characterization of the recombinant protein. Our results show that $O$. tauri SSIII-C isoform comprises an active enzyme that preferably uses ADPGlc as substrate and shows a structure of the active site region conserved respect to the SSIII from A. thaliana or bacterial GSs, such as the enzymes from E. coli or Agrobacterium tumefaciens. 


\section{Materials and methods}

\subsection{Strains and culture media}

Different commercial strains of E. coli from Stratagene (CA, USA) were used in this study. Specifically strains: XL1Blue (endA1, gyrA46, hsdR17, lac', recA1, relA1, supE44, thi-1, $\mathrm{F}^{\prime}\left[\right.$ proAB $^{+}$, lacI ${ }^{\mathrm{q}}$ lacZ $\Delta \mathrm{M} 15$, Tn10(tet $\left.\left.{ }^{\mathrm{r}}\right)\right]$ ) and E. coli BL21 (DE3) pLysS [FompT gal dcm $h s d S_{\mathrm{B}}\left(\mathrm{r}_{\mathrm{B}^{-}} \mathrm{m}_{\mathrm{B}^{-}}\right) \lambda(\mathrm{DE} 3)$ pLysS $\left.\left(\mathrm{Cm}^{\mathrm{r}}\right)\right]$. All the cells were grown at $37{ }^{\circ} \mathrm{C}$ in LB medium containing the proper antibiotics.

\subsection{Cloning, expression and purification of OsttaSSIII-C}

The gene OsttaSSIII-C (Ot06g03410) was cloned from O. tauri genomic DNA (kindly provide for Dr. Evelyne Derelle) into EcoRI and HindIII sites of pRSET-B (Invitrogen CA, USA), using standard molecular biology procedures and the primers: OtSSIII-C Nt Fwd, ACGAATTCGGACCAGCGCCCATC and OtSSIII-C Ct Rev: ATGAAGCTTCGTGAGACTACCG (EcoRI and HindIII sites underlined).

The expression vector pRSET-B::OsttaSSIII-C (containing an N-terminal His-tag sequence) was used to transform E. coli BL21(DE3) pLysS strain. Cells were grown at 37 ${ }^{\circ} \mathrm{C}$ for $3 \mathrm{~h}$, and then $1 \mathrm{mM}$ IPTG was added and incubated at $30{ }^{\circ} \mathrm{C}$ for at least $4 \mathrm{~h}$. Cells were harvested by centrifugation at $5000 \times \mathrm{g}$ for $15 \mathrm{~min}$ at $4{ }^{\circ} \mathrm{C}$. Pellet was suspended in buffer containing $20 \mathrm{mM}$ Tris- $\mathrm{HCl}(\mathrm{pH}$ 7.5). Cells were disrupted by sonication and centrifuged at $12000 \times \mathrm{g}$ for $15 \mathrm{~min}$ at $4{ }^{\circ} \mathrm{C}$. The homogenate was loaded onto a HiTrap chelating HP column (GE Healthcare BioSciences, Uppsala, Sweden) equilibrated with binding buffer (20 mM Tris-HCl, $\mathrm{pH} 7.5$, and $20 \mathrm{mM}$ imidazole). The column was washed with 10-15 volumes of binding buffer, and each protein was eluted using a linear gradient of binding buffer and elution buffer (20 mM Tris- $\mathrm{HCl}, \mathrm{pH} 7.5$, and 20-500 mM imidazole) [8]. The presence of OsttaSSIII-C $(56.8 \mathrm{kDa})$ in the eluted fractions was monitored by SDS-PAGE analysis. Fractions containing the protein of interest were concentrated to $>1$ mg/ml using Vivaspin 610000 MWCO concentrators (GE Healthcare BioSciences,

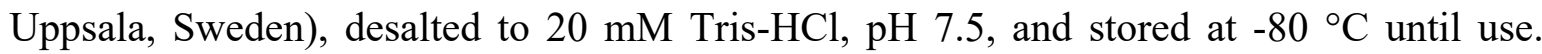
Under these conditions the purified enzyme remained fully active during at least three months. 


\subsection{Bioinformatic analysis}

Two approaches were used to detect domains in OsttaSSIII-C: the CD-search server [14] and the InterPRO resource [15]. Alignment of the OsttaSSIII-C, ArathSSIII-CD, AgrtuG, EsccoGS, ChlreSSIII1 CD and ChlreSSIII2 CD amino acids sequence were performed by using the Unipro UGENE v.1.10.4 program [16] with default parameters.

\subsection{Homology modelling}

The 3D structure modeling of OsttaSSIII-C and modified proteins, were performed with@TOME V2.2 server, which includes Scwrl, Modeller [17] and T.I.T.O. (Tool for Incremental Threading Optimisation). Structures were modeled using glycogen synthase from E. coli (PDB code 2QZS) as a template [18]. The alignment was based on homology and secondary structure. Models were evaluated using Verify-3D [19] and ProSA-web [20] programs.

\subsection{Construction, expression and purification of site directed mutated proteins.}

The mutated OsttaSSIII-C proteins R270A, K275A and E352A were obtained using the QuickChange II site-directed mutagenesis kit (Stratagene, CA, USA). The pRSETB::OsttaSSIII-C vector was used as the template for PCR amplification. The following primers (and their complements) were used (base substitutions in italics and underlined): OtC R270A, TGGCGTTGTCTCAGCCCTTACCGCTC; CTTACCGCTCAAGCAGGTATCCATCTC and OtC E352A, CCGTCAATGTTTGCGGCCGTGCGGTCTGAC. The mutated sequences were confirmed by DNA sequencing to control PCR fidelity (Macrogen, Korea). Recombinant plasmids named pRSET-B::OsttaSSIII-C R270A, pRSET-B::OsttaSSIII-C K275A and pRSETB::OsttaSSIII-C E352A (containing an N-terminal His-tag sequence) were used to transform E. coli BL21(DE3) pLysS cells. Mutated recombinant proteins were produced, purified and conserved as described above in item 2.2 for the wild type enzyme. 


\subsection{Determination of starch synthase activity}

Activity of O. tauri SSIII-C wild type and the mutated proteins was determined following the formation of Pi (after hydrolysis of generated ADP by alkaline phosphatase) by using a colorimetric assay adapted from $\mathrm{Wu}$ et al [21]. The reaction medium (50 $\mu 1$ final volume) contained $50 \mathrm{mM}$ Bicine, $\mathrm{pH} 8.0,1 \mathrm{U}$ of E. coli alkaline phosphatase (SigmaAldrich, MO, USA), 0-2 mg/ml of rabbit muscle glycogen or 0-4 mg/ml amylopectin, and 0-1 mM ADPGlc. When UDPGlc was used as the glycosyl donor, the assay conditions were identical, except that ADPGlc was replaced by UDPGlc in the range 0-10 mM. After incubation for $10 \mathrm{~min}$ at $35^{\circ} \mathrm{C}$ the reaction was stopped by the addition of Malachite Green reagent. The complex formed with the released Pi was measured at $630 \mathrm{~nm}$. Inorganic phosphate was used as standard. One unit of enzyme activity was defined as the amount of protein catalyzing the incorporation of $1 \mu \mathrm{mol}$ of Glc from ADPGlc into glycogen per 1 min, under the specified conditions.

The kinetic data were plotted as initial velocity ( $\mu \mathrm{mol} / \mathrm{min} . \mathrm{mg}$ protein) versus substrate concentration. The maximum catalytic rate and the Michaelis-Menten constants $\left(K_{\mathrm{m}}\right)$ for the binding of the substrates were determined from a non-linear fit of the Michaelis-Menten equation to the observed enzyme activities using GraphPad Prism version 6.01 for Windows (GraphPad Software, La Jolla California USA, www.graphpad.com). All kinetic parameters are the mean of at least three determinations and the average values $\pm \mathrm{SE}$ are reported. Total protein was determined using the Bradford method [22].

A pH profile of OsttaSSIII-C activity was conducted by measuring activity at $35^{\circ} \mathrm{C}$ and $\mathrm{pH}$ values ranging from 6.0 to 10.0 in $50 \mathrm{mM}$ Bicine buffer. The temperature profile of OsttaSSIII-C activity was followed by measuring activity at $\mathrm{pH} 8.0$ and temperature values ranging from 20 to $45{ }^{\circ} \mathrm{C}$ in $50 \mathrm{mM}$ Bicine buffer. Both experiments were run with 2 $\mathrm{mg} / \mathrm{ml}$ glycogen and $1 \mathrm{mM}$ ADPGlc. Standard kinetic parameters were determined at $\mathrm{pH}$ 8.0 and $35^{\circ} \mathrm{C}$. 


\subsection{Circular Dichroism}

Circular dichroism far-UV spectra were obtained using a Jasco J810 spectropolarimeter (Jasco Int. Co., Japan). Measurements were performed in a $0.1 \mathrm{~cm}$ quartz cuvette and the setting was as follows: measurement rate $40 \mathrm{~nm} \mathrm{~min}{ }^{-1}$, bandwidth 1 $\mathrm{nm}$, response time $2 \mathrm{~s}$, data pitch $1 \mathrm{~nm}$, and spectra accumulation of 15 . The range of wavelength used was from $250 \mathrm{~nm}$ to $200 \mathrm{~nm}$, at $20^{\circ} \mathrm{C}$. All spectra data are reported as molar ellipticity $[\theta]$ molar, $\lambda\left(\mathrm{deg} \mathrm{cm}^{2} \mathrm{dmol}^{-1}\right)$ obtained after subtracting the baseline, and smoothing data. 


\section{Results and discussion}

\subsection{Sequence analysis and homology modeling of OsttaSSIII-C}

The catalytic activity of SSs and GSs is conducted by a highly conserved region common between these enzymes [23]. This $60 \mathrm{kDa}$ "core" region is in the C-terminus of SSs from plant and algae, but it encompasses the entire protein sequence of bacterial GSs. An OsttaSSIII-C sequence analysis was done with CD-search and InterPro servers and the two typical SS domains, GT-5 (Pfam PF08323) and GT-1 (Pfam PF00534) were detected, with significantly low E-values $\left(3.0 \times 10^{-53}\right.$ and $4.5 \times 10^{-79}$, respectively) (Figure $\left.1 \mathrm{~A}\right)[14$, 15]. However, we could not identify any SBD characteristic of other SSIIIs within the amino acids sequence of the $56.8 \mathrm{kDa} O$. tauri protein, suggesting that OsttaSSIII-C has only a catalytic domain and it lacks of a SBD; which resembles the characteristic found in prokaryotic GSs.

Amino acid residues involved in substrates binding and catalysis has been identified in several SS and GS sequences. The alignment of OsttaSSIII-C, ArathSSIII-CD, EsccoGS and AgrtuGS amino acid sequences showed a high conservation of these residues in the $O$. tauri SSIII-C isoform (Figure 1 B, Table 1 and 2). It has been reported that Arg299 and Lys304 from A. tumefaciens are involved in the catalytic activity of GS, specifically in the interaction with a phosphate group of ADPGlc [23-25]. Glu376 is also important, making a salt bridge with Lys304 and helps to stabilize the glucose bound to ADP. In addition, it was also reported that these groups are conserved in E. coli (Arg300, Lys305 and Glu375) [18, 26] and A. thaliana SSIII (Arg837, Lys842 and Glu919) [27]. Accordingly, Figure 1 B showed a high conservation of these residues in the O. tauri SSIII-C isoform (Arg270, Lys275 and Glu352, OsttaSSIII-C numbering).

Using the 3D structure of the E. coli GS (PDB code 2QZS, 32\% identity for residues 1-465) as template, we built a homology model of OsttaSSIII-C as described in the Materials and Methods section. The structure of OsttaSSIII-C presents a folding similar to 2QZS, being secondary structure conserved along the sequence (Figure 2 A-B) and exhibiting a spatial conservation of the position of the amino acids involved in catalysis (Arg300, Lys305 and Glu375 for 2QZS numbering; Arg270, Lys275 and Glu352, 


\section{Figure 1}

A

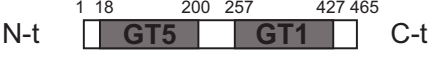 \\ OsttaSSIII-C}

B

OsttaSSIIIC 001 MDYHTVIVNGSGPAPILKVVHVAVEMAPIAKVGGMGDVVTALARATQEDGHQVEVFVPHYDIAQFENVDGYH 072 ArathSSIII CD 574 Th AgrtuGS $001-0105$ ESCCOGS 001 -

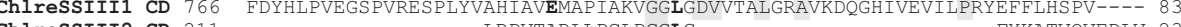
ChlreSSIII2 CD 211 ---------------LRPVTARLIPCLPGGLG------------------FYKATVQVEDLV 239

\begin{tabular}{|c|c|c|c|c|}
\hline & & & 112 & \\
\hline SIIIC & & 073 & & \\
\hline SIII & $\mathrm{CD}$ & 633 & FNRSYHWGG------TE IKVWHGKVEGLSVYFLDPQNGLFQRGCVYG- & \\
\hline grtuGs & & & LLILDAPAYYERSGGPYLGQTGKDYPDNWKRFAALSLAAARI & \\
\hline & & & $---G H I T L L F G H Y N G V G I Y L I D A P H L Y D R P G S P Y H D T N L F A Y T D N V$ & \\
\hline & $\mathrm{CD}$ & & FFATPTVYG------R & \\
\hline & $\mathrm{CD}$ & & CIYG-----KI & \\
\hline & & & $T H$ & \\
\hline 2 & $\mathrm{CD}$ & 6 & G-- - & \\
\hline & & & 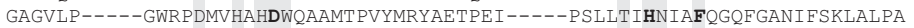 & \\
\hline isc & & & AK------SVFTVHNLAYQGMFYAHHMNDIQLPW & \\
\hline & & & 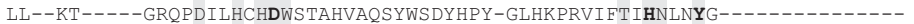 & \\
\hline SSIII2 & & & ADI I HCHDWQSAPVAYG-. & \\
\hline
\end{tabular}

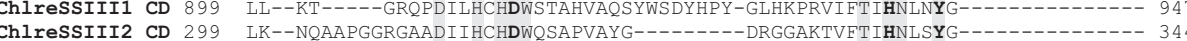

OsttaSSIIIC 173 ------------VDRIRRGMESCDIATTVSPTYADEVRFHHAIA---------PSKDKFIGIRNGIDTDIWN 223 ArtathSSIII CD 741 ---------ANAIGKAMTFADKATTVSPTYAKEVAGNSVIS--------AHLYKFHGIINGIDPDIWD 791 $\begin{array}{lrl}\text { ArathSSIII } & \mathrm{CD} & 741 \\ \text { AgrtuGS } & 184 \text { HAFGMEGIEYYNDVSFLKGGLQTATALSTVSPSYAEEILTAEFGMGLEGVIGS--RAHVLHGIVNGIDADVWN } 255\end{array}$

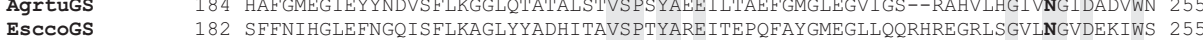

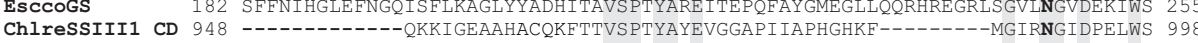
ChlreSSIII2 CD 345 ----------ADLIGRAMQACQVATTVSPTYAREIAGHPSVAAHLNKL--------YGVLNGIDQDIWD 395

OsttaSSIIIC 224 PANDKFLPVGYNRSNAIDGKRAAAAELCNRLGLEHPEGSPIVGVVSRTTA 275 KIHLIKHACYRVLERGATFVL 296 ArathSSIII CD 792 PYNDNFIPVPYTSENVVEGKRAAKEELQNRLGLKS-ADFPVVGIIIRETHOKGIHLIKHAIWRTLERNGQVVL 863 AgrtuGS 256 PATDHLIHDNYSAAN-LKNRALNKKAVAEHFRID-DDGSPLFCVI RETWDKGIDLMAEAVDEIVSLGGRLVV 328 256 OTDIT 256 ChlreSSIII2 CD 396 PSEDPCLPLHFSADNVAAGKEAARRALRQKLGLVAAD-VPIVGCVIR TVAOKGIHLSKHAAWRTLERGGQFVL 467

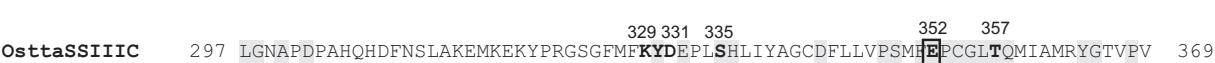
ArathSSIII CD 864 LGSAPDPRIQNDFVNLANQLHSSHGDRARLVLTYDEPLSHLIYAGADFILVPSI EECGLTQLIAMRYGAVPV 936 AgrtuGS $\quad 329$ LG-AGDVALEGALLAAASRHHG----RVGVAIGYNEPLSHLMQAGCDAIIIPSRAEPCGLTQLYALRYGCIPV 397 EscCOGS $\quad 330$ LG-AGDPVLQEGFLAAAAEYPG----QVGVQIGYHEAFSHRIMGGADVILVPSR EPCGLTQLYGLKYGTLPL 398 ChlreSSIII1CD 1072 LGSAPDPKVQADFNALAQSMGGQDAAFC---FKYDEPLSHLIYAAADMIVVPSMEEPCGLTQMIAMRYGTVPI 1141 ChlreSSIII2CD 468 LGSAPDGRVQGEFNALREQLSRAYPDRAALVFTYDEPLSHLIYAGSDMFLVPSMEEPCGLTQMIAMRYGTIPV 540

OsttaSSIIIC 370 VRRTGGLADTVFDVENDSTRCATAGIPP--------_--NGFVFDGTETRDIDSAVIRALDAFYDRERWESLA 431 ArathSSIII CD 937 VRKTGGLFDTVFDVDHDKERAOAOVLEP-_-_-_-_-_NGFSFDGADAPGVDYALNRAISAWYDGREWF-NS 997 AgrtuGS 398 VARTGGLADTVIDA--NHAALASKAAT---_-_-_----TGVQFSPVTLDGLKOAIRRTVRYYHDPKLWT--0 455 EscCOGS 399 VRRTGGLADTVSDC--SLENLADGVA----------SGFVFEDSNAWSLLRAIRRAFVLWSRPSLWR--F 455 ChlreSSIII1CD 1142 VRHTGGLRDTVFDVDFDKERAAWELYGSSDWQRDGIDATNGFAFTGTDGGALDYAMNRAIDAWYNDRAWF-HS 1213 ChlreSSIII2CD 541 VRKTGGLVDTVYDVDHDEDRARLKGMDT---------NGFSFEGTDFAGMDYALNRALSVWYSDKALWHSL 602

OsttaSSIIIC 432 LVDRAMSCDWGWFEPSKRYEDLYWSALSKKRNTR 465

ArathSSIII CD 998 LCKTVMEODWSWNRPALEYLELYHSARK------ 1025

\begin{tabular}{lll} 
AgrtuGS & 456 MQKLGMKSDVSWEKSAGLYAALYSQLISKGH--- & 48 \\
\hline & 456 & VQROAMAMDFSWQVAAKSYREIYYRIK------
\end{tabular}

ChlreSSIIIICD 1214 IORRTMEQDWSWNRPATDYTEYFSAIKA------ 1242

ChlreSSIII2CD 603 RRKRAMTQDWSWNSPALDYVELYYKALKS----- 631 
Fig. 1. (A) Schematic representation of OsttaSSIII-C. GT1 and GT5 domain are shown. (B) Alignment obtained using the Unipro UGENE v.1.10.4 program (with default parameters) between OsttaSSIII-C, ArathSSIII-CD (Arabidopsis thaliana SSIII catalytic domain), AgrtuGS (Agrobacterium tumefaciens glycogen synthase), EsccoGS (Escherichia coli glycogen synthase), ChlreSSIII1 CD and ChlreSSIII2 CD (Chlamydomonas reinhardtii SSIII 1 and 2, catalytic domains). OsttaSSIII-C amino acids residues R270, K275 and E352 are indicated by open boxes (corresponding to site active amino acids R300, K305 and E377 characterized in EsccoGS [18, 26]). Other previously characterized catalytic residues are shown in bold. Grey boxes indicate identical residues. 
Table 1. Amino acid residues involved in ADPGlc binding in E. coli GS and OsttaSSIII-C

Ref: AthSSIII: Arabidopsis thaliana SSIII isoform; Adenine, ribose and Pi (phosphate) indicate which part of the Glc bound to ADP participates in the each interaction; $\mathrm{O} 2$ and O6: oxygen atoms in ribose; vdW: van der Waals interaction; KTGGM and KVGGL correspond to the conserved motifs in E. coli GS and OsttaSSIII-C enzymes, respectively.

\begin{tabular}{lllll}
\hline E. coli GS & OsttaSSIII-C & AthSSIII & Function & Conservation \\
\hline Lys15 & Lys31 & Lys591 & ADPGlc binding (KTGGM/KVGGL) & + \\
Gly18 & Gly34 & Gly594 & ADPGlc binding (KTGGM/KVGGL) & + \\
His161 & His167 & His736 & H bond with O6 ribose (380 loop) & + \\
Asn246 & Asn215 & Asn783 & H bond with O6 ribose & + \\
Val297 & Val268 & Ile835 & H bond with O2 ribose & + \\
Ser298 & Ser269 & Thr836 & vdW with adenine & + \\
Arg300 & Arg270 & Arg837 & ionic interaction with Pi & + \\
Lys305 & Lys275 & Lys842 & ionic interaction with Pi & + \\
Gly354 & Lys329 & Thr896 & interaction with adenine & + \\
Tyr355 & Tyr330 & Tyr897 & stacking with adenine & - \\
His356 & Asp331 & Asp898 & carbonyl interacts with adenine & + \\
Ser360 & Ser335 & Ser902 & vdW with adenine & + \\
Glu377 & Glu352 & Glu919 & ionic interaction with Pi & + \\
Thr382 & Thr357 & Thr924 & H bond with O2 ribose & + \\
& & & & + \\
\hline
\end{tabular}


Table 2. Amino acid residues involved in glycogen binding in E. coli GS and OsttaSSIII-C

Ref: Arabidopsis thaliana SSIII isoform; Glc +1 and Glc +2 indicates which Glc in glycogen is involved in the interaction; $\mathrm{OH} 2$ and $\mathrm{OH} 3$ indicates $\mathrm{OH}$ groups of Glc +1 that interacts with the protein; KTGGL and KVGGM correspond to the conserved motifs in E. coli GS and OsttaSSIII-C enzymes, respectively.

\begin{tabular}{llllc}
\hline E. coli GS & OsttaSSIII-C & AthSSIII & Function & Conservation \\
\hline Glu9 & Glu25 & Glu585 & binding of Glc +1 & + \\
Leu19 & Met35 & Leu595 & binding of Glc +1 (KTGGL/KVGGM) & - \\
Tyr 95 & Tyr112 & Tyr672 & stacking with Glc +2 & + \\
Asp137 & Asp146 & Asp706 & H bond with OH2 and OH3 Glc +1 & + \\
His161 & His167 & His736 & H bond with O6 ribose (380 loop) & + \\
Tyr165 & Phe171 & Phe740 & stacking with Glc +2 & + \\
Arg299 & Arg270 & Arg837 & ionic interaction with Pi & + \\
& & & & \\
\hline
\end{tabular}


Figure 2
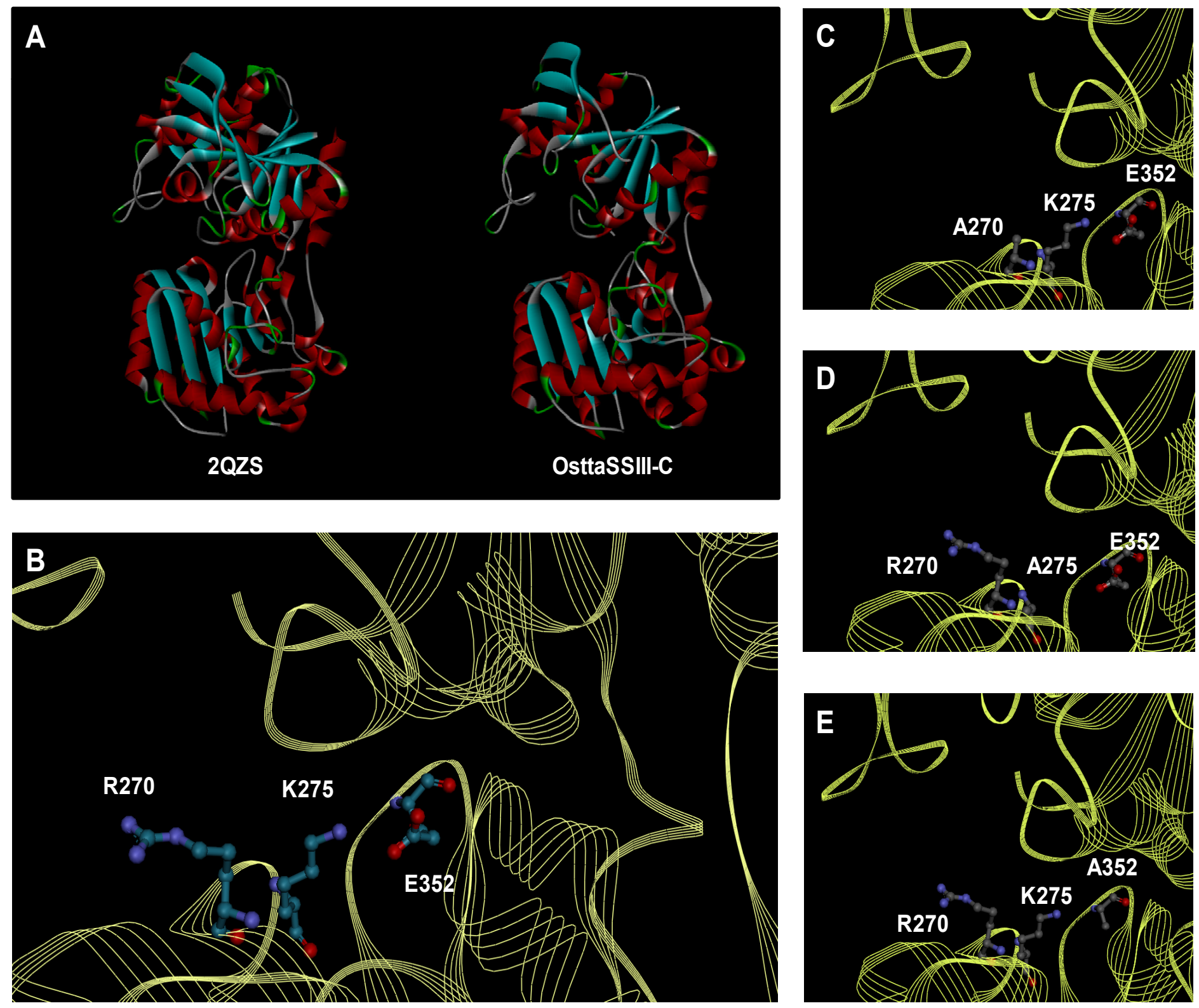
Fig. 2. Homology modelling of OsttaSSIII-C and mutants. (A) Structural model of E. coli GlgA (PDB 2QZS, left) and the proposed model for OsttaSSIII-C (right); (B) Close-up view of catalytic site of OsttaSSIII-C, showing the residues involved in the active site; (C, D, E) Close-up view of catalytic site of OsttaSSIII-C, showing the residues involved in the active site for the mutants R270, K275 and E352 respectively. 
OsttaSSIII-C numbering, see Figure 2), suggesting that also in this protein such residues would have an important role in catalysis.

\subsection{Cloning, expression and purification of OsttaSSIII-C wt and modified proteins.}

Three genes coding for respective isoforms of SSIII have been identified in O. tauri. We focused our attention on OsttaSSIII-C, where the lack of an SBD makes it more similar to prokaryotic GSs. The DNA fragment coding for OsttaSSIII-C was cloned into pRSET-B vector to generate the pRSET-B::OsttaSSIII-C plasmid. This construct leads to the expression of OsttaSSIII-C with the His-tag at the N-terminal region of the protein. To test the importance of the amino acid residues Arg270, Lys275 and Glu352 in OsttaSSIII-C catalysis, the modified proteins OsttaSSIII-C R270A, K275A and E352A were obtained after performing site directed mutagenesis on pRSET-B::OsttaSSIII-C (see Materials and Methods). The purification of OsttaSSIII-C wt and mutated proteins were carried out through a single step using HiTrapTM Chelating HP column equilibrated with $0.1 \mathrm{M}$ $\mathrm{NiSO}_{4}$. Using this procedure, we obtained about 1-1.5 mg of purified SSIII-C per gram of cells. Figure 3 (lane 2) shows the SDS-PAGE profile of recombinant OsttaSSIII-C wt. The purification analysis of the modified proteins is shown in lanes 3 to 5 . Results show a single

protein band in all the lanes from the SDS-PAGE with an estimated molecular mass of $\sim 57$ $\mathrm{kDa}$ with no degradation products.

\subsection{Kinetic characterization of OsttaSSIII-C.}

The kinetic parameters of OsttaSSIII-C were determined using the purified recombinant protein expressed in E. coli cells. Figure 4 shows the saturation plots for the enzymatic activity of OsttaSSIII-C wt using ADPGlc as the sugar-nucleotide donor (Figure $4 \mathrm{~A}$ ) and glycogen (Figure $4 \mathrm{~B}$ ), or amylopectin (Figure $4 \mathrm{C}$ ) as the acceptor substrate. In all cases the OsttaSSIII-C enzyme displayed Michaelis kinetics, indicating a noncooperative behavior. Table 3 shows the kinetic parameters of OsttaSSIII-C in the assayed conditions. The $K_{\mathrm{m}}$ values for the acceptor polysaccharide were $0.23 \mathrm{mg} / \mathrm{ml}$ for glycogen and $0.64 \mathrm{mg} / \mathrm{ml}$ for amylopectin; whereas the respective value for ADPGlc was $99 \mu \mathrm{M}$, using glycogen as the acceptor substrate. These $K_{\mathrm{m}}$ values are in the same range as those reported for SS isoenzymes from other plants and algae, including Chlamydomonas 


\section{Figure 3}

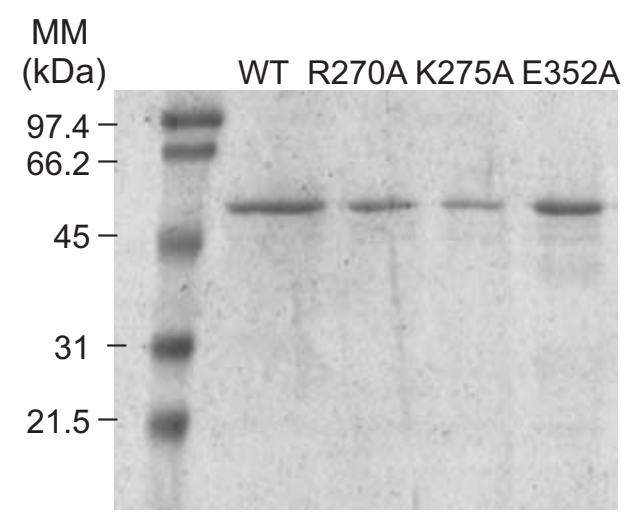

Fig. 3. (A) SDS-PAGE of recombinant enzymes. Lane 1: Molecular weight standard, lane 2, OsttaSSIII-C; lane 3, OsttaSSIII-C R270A; lane 4, OsttaSSIII-C K275A; and lane 5, OsttaSSIII-C E352A. Numerals indicate the molecular masses (MM) of the standards (Prestained SDS-PAGE Standards, Low Range, BioRad). 
Figure 4
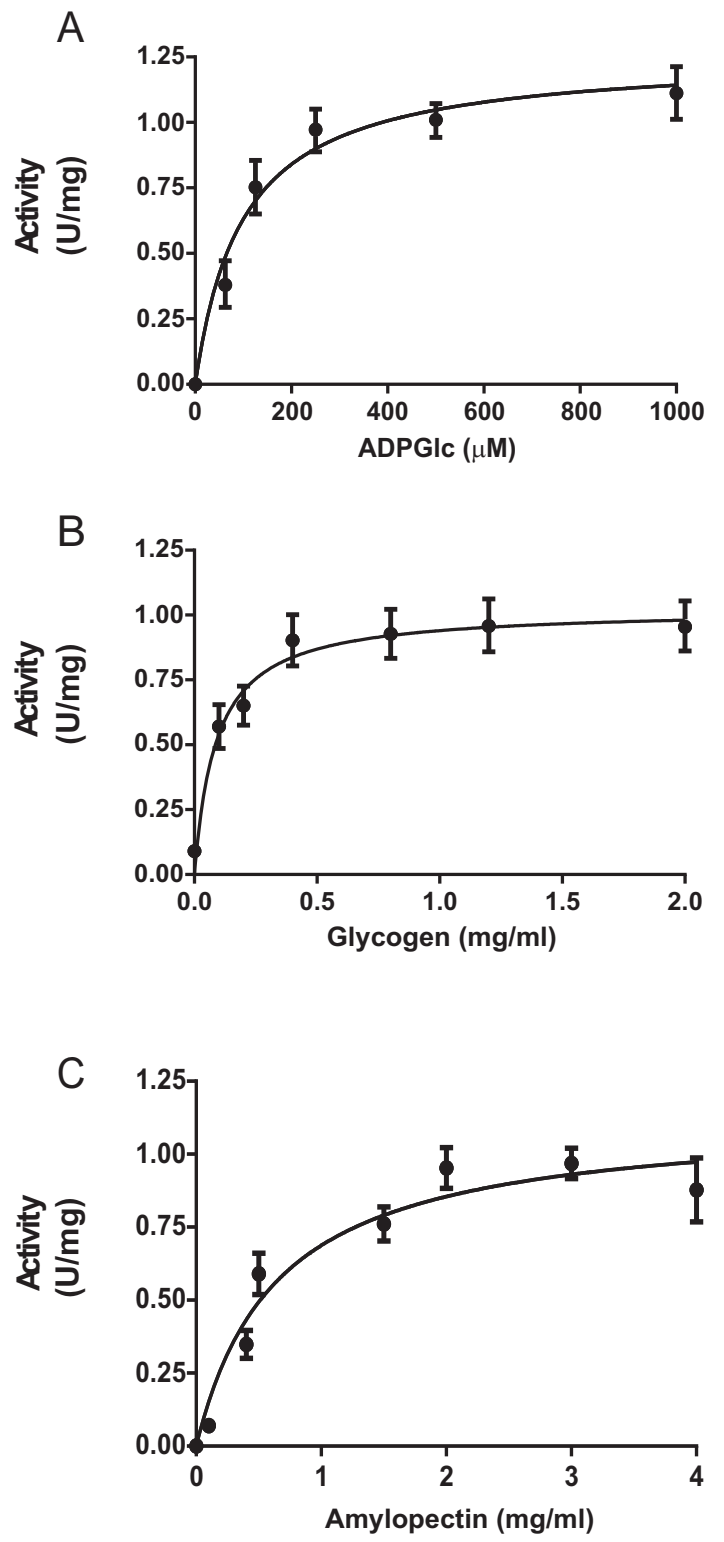

Fig. 4. ADPGlc (A), glycogen (B) and amylopectin (C) saturation plots for OsttaSSIII$\mathrm{C}$ determined in the presence of $2 \mathrm{mg} / \mathrm{ml}$ glycogen (A) or $500 \mu \mathrm{M}$ ADPGlc (B and C). 
Table 3. Kinetic parameters of wild type OsttaSSIII-C

\begin{tabular}{llc}
\hline & $\begin{array}{c}K_{\mathrm{m}} \\
(\text { ADPGlc } \mathrm{mM} \\
\text { Polysacc } \mathrm{mg} / \mathrm{ml})\end{array}$ & $\begin{array}{c}V_{\max } \\
(\mathrm{U} / \mathrm{mg})\end{array}$ \\
\hline ADPGlc & $0.099 \pm 0.008$ & $1.25 \pm 0.03$ \\
Glycogen & $0.23 \pm 0.02$ & $1.14 \pm 0.06$ \\
Amylopectin & $0.64 \pm 0.04$ & $1.09 \pm 0.05$ \\
Amylose & n.a. & n.a. \\
\hline n.a., no activity & &
\end{tabular}

n.a., no activity 
reinhardtii $[8,28,29]$. As mentioned above, C. reinhardtii presents two starch synthases III [30], containing two and three SBDs [6], that are structurally more similar to OsttaSSIII-A, OsttaSSIII-B, and ArathSSIII rather than OsttaSSIII-C. However, to date, only one $C$. reinhardtii SSIII isoform was kinetically characterized (ChlreSSIII1, Genbank AAY42381.1), showing kinetic parameters in the same range to those reported for OsttaSSIII-C [28].

Under the assayed conditions OsttaSSIII-C did not exhibit enzymatic activity when amylose and UDPGlc were used as acceptor and donor substrates, respectively. These results reinforced the previous statement that $O$. tauri synthesize starch through the ADPGlc pathway [7]. In addition, like other plants and algae starch synthases, OsttaSSIII-C showed an alkaline $\mathrm{pH}$ optimum close to 8 , in Bicine buffer, and an optimum reaction temperature of $35^{\circ} \mathrm{C}$ (Figure $5 \mathrm{~A}$ and $\mathrm{B}$ ). On the other hand, we analyzed the effect of different metabolites such as citrate and malate. It was previously reported that both metabolites act as activators, increasing the SS activity in maize and rice [31, 32]. However, no effect on OsttaSSIII-C activity was observed after the addition of different concentrations of malate or citrate (0 to $500 \mathrm{mM})$.

\subsection{Effect of R270, K275 and E352 on OsttaSSIII-C catalytic activity.}

To test the importance of the amino acid residues Arg270, Lys275 and Glu352 in catalysis, the kinetic parameters of each isoform were determined as described in Materials and Methods. The results are shown in Table 4. All three residues mutated in this analysis were critical for catalysis, as the replacement of any of them decreased the specific activity several orders of magnitude. Replacement of Arg270 or Lys275 by Ala rendered an enzyme about 301/327- and 52/74-fold less active regards ADPGlc and glycogen, respectively. When Glu352 was mutated, the catalytic efficiency decreased 146- and 14-fold for ADPGlc and glycogen, respectively. We ruled out the possibility that the mutations generated misfolded forms of the enzyme, because their circular dichroism spectra were near identical (Figure 6), suggesting that these mutations did not affect enzyme structure. Our results indicate that these residues play an important role in the catalytic reaction, and suggest that 
Figure 5
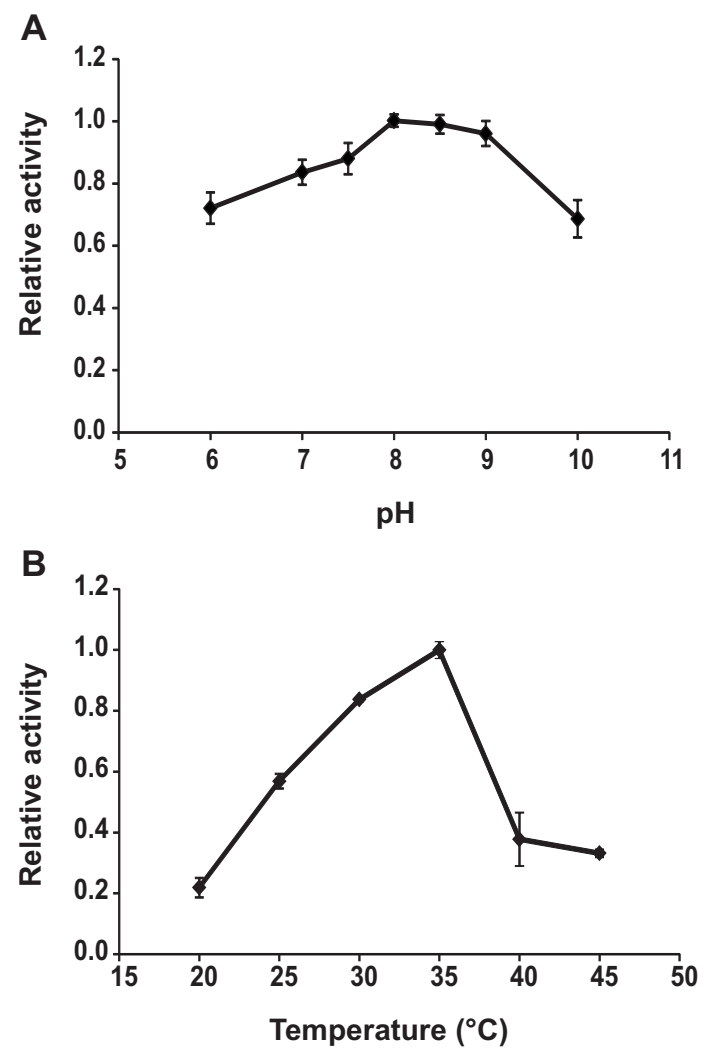

Fig. 5. (A) Effect of $\mathrm{pH}$ on the activity of OsttaSSIII-C. $50 \mathrm{mM}$ Bicine buffer was used for $\mathrm{pH}$ 6.0-10.0. Assays were run with $2 \mathrm{mg} / \mathrm{ml}$ glycogen and $1 \mathrm{mM}$ ADPGlc. Data are relative values compared with activity at $\mathrm{pH}$ 8. (B) Effect of temperature on the activity of OsttaSSIII-C. Assays were run with $2 \mathrm{mg} / \mathrm{ml}$ glycogen and $1 \mathrm{mM}$ ADPGlc, in 50 $\mathrm{mM}$ Bicine buffer, with reaction temperatures of $20,25,30,35,40$ and $45^{\circ} \mathrm{C}$. Data are relative values compared with activity at $35^{\circ} \mathrm{C}$. 


\section{Figure 6}

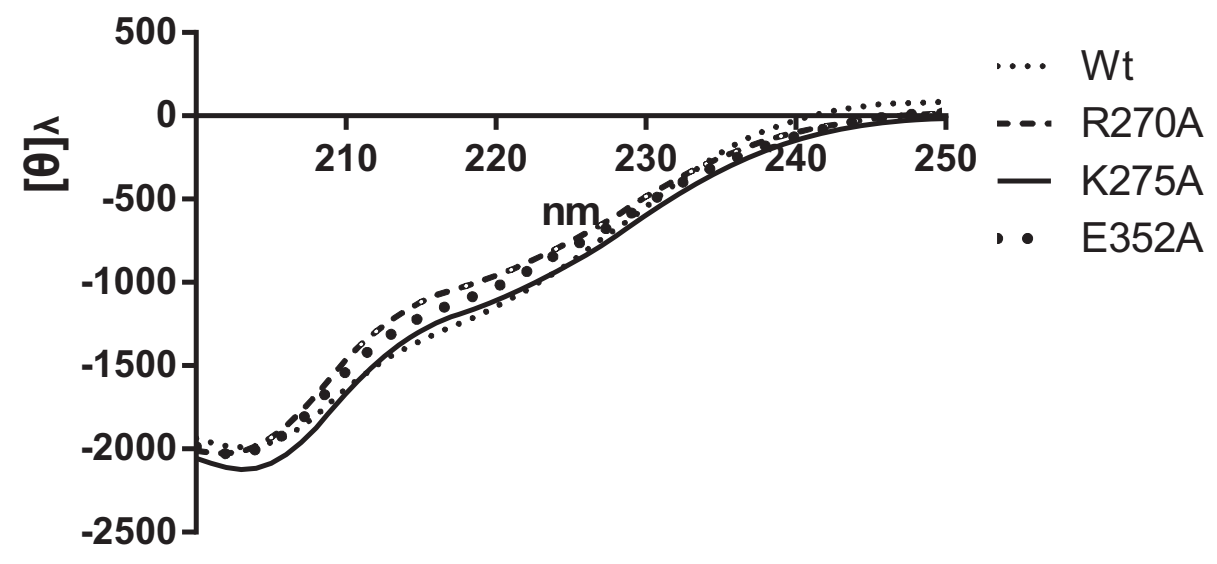

Fig. 6. Far-UV Circular Dichroism Spectra of recombinant and mutated OsttaSSIII-C isoforms. Wild type protein is represented by dotted lines. OsttaSSIII-C R270A, K275A and $\mathrm{E} 352 \mathrm{~A}$ are represented by dashed line, solid line and bold dotted line, respectively. Circular Dichroism spectra were obtained in $20 \mathrm{mM}$ Tris-HCl buffer, $\mathrm{pH}$ : 7.5. 
Table 4. Kinetic parameters of wild type OsttaSSIII-C and modified proteins

\begin{tabular}{|c|c|c|c|c|c|c|c|c|}
\hline & \multicolumn{4}{|c|}{ ADPGlc } & \multicolumn{4}{|c|}{ glycogen } \\
\hline & $\begin{array}{l}K_{\mathrm{m}} \\
(\mathrm{mM})\end{array}$ & $\begin{array}{c}V_{\max } \\
\left(\mathrm{mU} \cdot \mathrm{mg}^{-1}\right)\end{array}$ & $\begin{array}{c}V_{\max } / K_{\mathrm{m}} \\
\left(\mathrm{mU} \cdot \mathrm{mg}^{-1} \cdot \mathrm{mM}^{-1}\right)\end{array}$ & $\begin{array}{l}\text { Catalytic } \\
\text { efficiency (\%) }\end{array}$ & $\begin{array}{l}K_{\mathrm{m}} \\
\left(\mathrm{mg} \cdot \mathrm{ml}^{-1}\right)\end{array}$ & $\begin{array}{l}V_{\max } \\
\left(\mathrm{mU} \cdot \mathrm{mg}^{-1}\right)\end{array}$ & $\begin{array}{c}V_{\max } / K_{\mathrm{m}} \\
\left(\mathrm{mU} \cdot \mathrm{mg}^{-1}\right) \cdot\left(\mathrm{mg} \cdot \mathrm{ml}^{-1}\right)^{-1}\end{array}$ & $\begin{array}{l}\text { Catalytic } \\
\text { efficiency (\%) }\end{array}$ \\
\hline$\overline{\mathrm{WT}}$ & $0.10 \pm 0.01$ & $1230 \pm 110$ & $12300 \pm 2330$ & 100 & $0.24 \pm 0.03$ & $1176 \pm 97$ & $4900.0 \pm 612.6$ & 100 \\
\hline R270A & $0.13 \pm 0.02$ & $5.3 \pm 0.4$ & $40.8 \pm 3.2$ & 0.33 & $0.07 \pm 0.01$ & $6.6 \pm 0.7$ & $94.3 \pm 13.6$ & 1.9 \\
\hline $\mathrm{K} 275 \mathrm{~A}$ & $0.67 \pm 0.07$ & $25.2 \pm 3.1$ & $37.6 \pm 8.5$ & 0.31 & $0.44 \pm 0.05$ & $29.2 \pm 3.1$ & $66.4 \pm 7.6$ & 1.4 \\
\hline E352A & $0.25 \pm 0.04$ & $21.0 \pm 2.6$ & $84.0 \pm 10.6$ & 0.68 & $0.04 \pm 0.01$ & $14.0 \pm 2.3$ & $350.0 \pm 57.8$ & 7.1 \\
\hline
\end{tabular}


OsttaSSIII-C has a reaction mechanism similar to E. coli GS and other retaining GT-B glycosyltransferases $[18,26]$.

\section{Conclusions}

In the last years, there has been an increasing demand for starch in many industrial processes, such as pharmaceutical, food, and bioethanol production. Therefore, a better understanding of starch metabolism, in particular the structure-function relationship of the enzymes involved in its biosynthesis, may provide a powerful instrument for the development of new strategies to increase plant biomass as well as to improve the quantity and quality of this polysaccharide [33, 34]. O. tauri, a unicellular green alga of the Prasinophyceae family, has a very simple cellular organization and presents a single starch granule at the center of its unique chloroplast [7]. However, its starch metabolism exhibits a structural complexity comparable to higher plants, with multiple enzyme forms for each pathway step [35].

O. tauri possesses three SSIII isoforms, one similar to higher plants and Chlamydomonas SSIII, with three N-terminal SBDs (OsttaSSIII-B) and the other two isoforms, one with two central SBDs (OsttaSSIII-A) and the third one, lacking SBDs (OsttaSSIII-C) [6]. In this work we report the cloning, expression in E. coli cells and characterization of the SSIII-C isoform from this picoalga. We show that in vitro OsttaSSIII-C presents starch synthase activity. We evaluated its acceptor substrate specificity determining that OsttaSSIII-C displayed high ability to elongate branched glucans such as amylopecin and glycogen, whereas it showed inefficient to use amylose as the glucan acceptor. This agrees with the higher activity showed by rice SSI, SSIIa and SSIIIa [32], maize endosperm SSI, SSIIa, and SSIIb [36], potato tuber SSII [37], kidney bean SSIII [38] and pea embryo SSII [39], in the presence of glycogen and amylopectin with respect to maltohexaose. Furthermore, our results show that OsttaSSIII-C, which lacks SBDs, has the same substrate preference as the plant starch synthases containing SBDs. As previously reported, the main function of SBDs is related to the modulation of the 
biological activity of starch synthase III, rather than to determine the polysaccharide specificity [12].

On the other hand, OsttaSSIII-C displayed a high apparent affinity for ADPGlc, but it was not active when UDPGlc was used as the glucosyl donor substrate; agreeing a previous report that $O$. tauri starch metabolism is an ADPGlc based pathway [7].\#The OsttaSSIII-C N-terminal region would be involved in the interaction with ADPGlc, through the Lys-X-Gly-Gly-Leu motif [40]. This motif is strictly conserved in all the members of the SSIII family, where the variable X position is occupied by Val (Val32 in O. tauri). The amino acid residue Lys as well as Gly are conserved in the whole group in agreement with the fact that these residues are very important for the binding activity of the motif [41]. Furthermore, the Leu residue present in E. coli, A. tumefaciens and A. thaliana glycogen/starch synthase enzymes is replaced by a methionine in a conservative substitution for binding to glycogen. This change is also found in other algae such as Micromonas commoda, Micromonas pussilla, Volvox carteri and Ostreococcus lucimarinus (data not shown).

OsttaSSIII-C sequence alignment with SSIII-CD and glycogen synthases previously characterized $[18,24,26]$, identified conserved residues, and some of them were studied by site-directed mutagenesis. The kinetic characterization of the OsttaSSIII-C individual mutants in Arg270, Lys275, and Glu352 revealed the importance of the replaced residues. In all cases, the mutations significantly decreased the catalytic efficiency without affecting the protein folding. These results indicate that these residues play an important role in the catalytic reaction. Accordingly, this triad of residues has been described as critical for catalysis not only in glycogen/starch synthases but also in all other retaining GT-B glycosyltransferases such as the E. coli trehalose-6-phosphate synthase and maltodextrin phosphorylase [42] and also the sucrose synthases from A. thaliana and N. europaea [21, 43]. The conservation of the mentioned residues in OsttaSSIII-C and both SSIII isoforms from C. reinhardtii respect to all other retaining GT-B glycosyltransferases suggests that these enzymes would have the same reaction mechanism (Figure 1B).

In conclusion, our results support that the OsttaSSIII-C isoform is an active starch synthase enzyme without a N-terminal SBD. Further investigation is necessary to analyze 
the implications of the evolutionary conservation of this "bacterial like" starch synthase isoform in O. tauri and its physiological function in the picoalga starch metabolism.

\section{Acknowledgments}

We thank Dr. Evelyne Derelle for kindly providing O. tauri genomic DNA.

\section{Funding}

This work was supported by the Consejo Nacional de Investigaciones Científicas y Técnicas (CONICET, PIP 00134), Agencia Nacional de Promoción Científica y Tecnológica (ANPCyT, PICT RAICES 2011-0982; PICT Joven 2012-0981; PICT 20142436), and National Science Foundation (NSF MCB 1616851 and NSF MCB 1024945). $\mathrm{NH}$ is a doctoral fellow from ANPCyT. JB, AAI, MVB and DGC are research members from CONICET. 


\section{References}

1. Ball, S. G. \& Morell, M. K. (2003) From bacterial glycogen to starch: understanding the biogenesis of the plant starch granule, Annual review of plant biology. 54, 207-33.

2. Liu, H., Yu, G., Wei, B., Wang, Y., Zhang, J., Hu, Y., Liu, Y., Zhang, H. \& Huang, Y. (2015) Identification and Phylogenetic Analysis of a Novel Starch Synthase in Maize, Frontiers in plant science. 6, 1013.

3. Lombard, V., Golaconda Ramulu, H., Drula, E., Coutinho, P. M. \& Henrissat, B. (2014) The carbohydrate-active enzymes database (CAZy) in 2013, Nucleic acids research. 42, D490-5.

4. Ball, S., Colleoni, C., Cenci, U., Raj, J. N. \& Tirtiaux, C. (2011) The evolution of glycogen and starch metabolism in eukaryotes gives molecular clues to understand the establishment of plastid endosymbiosis, Journal of experimental botany. 62, 1775-801.

5. Cenci, U., Nitschke, F., Steup, M., Minassian, B. A., Colleoni, C. \& Ball, S. G. (2014) Transition from glycogen to starch metabolism in Archaeplastida, Trends in plant science. 19, 18-28.

6. Barchiesi, J., Hedin, N., Gomez-Casati, D. F., Ballicora, M. A. \& Busi, M. V. (2015) Functional demonstrations of starch binding domains present in Ostreococcus tauri starch synthases isoforms, BMC research notes. 8, 613.

7. Ral, J. P., Derelle, E., Ferraz, C., Wattebled, F., Farinas, B., Corellou, F., Buleon, A., Slomianny, M. C., Delvalle, D., d'Hulst, C., Rombauts, S., Moreau, H. \& Ball, S. (2004) Starch division and partitioning. A mechanism for granule propagation and maintenance in the picophytoplanktonic green alga Ostreococcus tauri, Plant physiology. 136, 3333-40.

8. Valdez, H. A., Busi, M. V., Wayllace, N. Z., Parisi, G., Ugalde, R. A. \& Gomez-Casati, D. F. (2008) Role of the N-Terminal Starch-Binding Domains in the Kinetic Properties of Starch Synthase III from Arabidopsis thaliana $\uparrow$, Biochemistry. 47, 3026-3032.

9. Martin, M., Wayllace, N. Z., Valdez, H. A., Gomez-Casati, D. F. \& Busi, M. V. (2013) Improving the glycosyltransferase activity of Agrobacterium tumefaciens glycogen synthase by fusion of N-terminal starch binding domains (SBDs), Biochimie. 95, 1865-70.

10. Palopoli, N., Busi, M. V., Fornasari, M. S., Gomez-Casati, D., Ugalde, R. \& Parisi, G. (2006) Starch-synthase III family encodes a tandem of three starch-binding domains, Proteins. 65, 27-31.

11. Valdez, H. A., Peralta, D. A., Wayllace, N. Z., Grisolía, M. J., Gomez-Casati, D. F. \& Busi, M. V. (2011) Preferential binding of SBD from Arabidopsis thaliana SSIII to polysaccharides: Study of amino acid residues involved, Starch - Stärke. 63, 451-460.

12. Wayllace, N. Z., Valdez, H. A., Ugalde, R. A., Busi, M. V. \& Gomez-Casati, D. F. (2010) The starch-binding capacity of the noncatalytic SBD2 region and the interaction between the $\mathrm{N}$ - and C-terminal domains are involved in the modulation of the activity of starch synthase III from Arabidopsis thaliana, The FEBS journal. 277, 428-40.

13. Gomez-Casati, D. F., Martin, M. \& Busi, M. V. (2013) Polysaccharide-synthesizing glycosyltransferases and carbohydrate binding modules: the case of starch synthase III, Protein and peptide letters. 20, 856-63.

14. Marchler-Bauer, A. \& Bryant, S. H. (2004) CD-Search: protein domain annotations on the fly, Nucleic acids research. 32, W327-31.

15. Hunter, S., Jones, P., Mitchell, A., Apweiler, R., Attwood, T. K., Bateman, A., Bernard, T., Binns, D., Bork, P., Burge, S., de Castro, E., Coggill, P., Corbett, M., Das, U., 
Daugherty, L., Duquenne, L., Finn, R. D., Fraser, M., Gough, J., Haft, D., Hulo, N., Kahn, D., Kelly, E., Letunic, I., Lonsdale, D., Lopez, R., Madera, M., Maslen, J., McAnulla, C., McDowall, J., McMenamin, C., Mi, H., Mutowo-Muellenet, P., Mulder, N., Natale, D., Orengo, C., Pesseat, S., Punta, M., Quinn, A. F., Rivoire, C., Sangrador-Vegas, A., Selengut, J. D., Sigrist, C. J., Scheremetjew, M., Tate, J., Thimmajanarthanan, M., Thomas, P. D., Wu, C. H., Yeats, C. \& Yong, S. Y. (2012) InterPro in 2011: new developments in the family and domain prediction database, Nucleic acids research. 40, D306-12.

16. Okonechnikov, K., Golosova, O. \& Fursov, M. (2012) Unipro UGENE: a unified bioinformatics toolkit, Bioinformatics. 28, 1166-7.

17. Pons, J. L. \& Labesse, G. (2009) @TOME-2: a new pipeline for comparative modeling of protein-ligand complexes, Nucleic acids research. 37, W485-91.

18. Sheng, F., Jia, X., Yep, A., Preiss, J. \& Geiger, J. H. (2009) The crystal structures of the open and catalytically competent closed conformation of Escherichia coli glycogen synthase, The Journal of biological chemistry. 284, 17796-807.

19. Luthy, R., Bowie, J. U. \& Eisenberg, D. (1992) Assessment of protein models with three-dimensional profiles, Nature. 356, 83-5.

20. Wiederstein, M. \& Sippl, M. J. (2007) ProSA-web: interactive web service for the recognition of errors in three-dimensional structures of proteins, Nucleic acids research. $\mathbf{3 5}$, W407-10.

21. Wu, Z. L., Ethen, C. M., Prather, B., Machacek, M. \& Jiang, W. (2011) Universal phosphatase-coupled glycosyltransferase assay, Glycobiology. 21, 727-33.

22. Bradford, M. M. (1976) A rapid and sensitive method for the quantitation of microgram quantities of protein utilizing the principle of protein-dye binding, Analytical biochemistry. 72, 248-54.

23. Leterrier, M., Holappa, L. D., Broglie, K. E. \& Beckles, D. M. (2008) Cloning, characterisation and comparative analysis of a starch synthase IV gene in wheat: functional and evolutionary implications, BMC plant biology. 8, 98.

24. Buschiazzo, A., Ugalde, J. E., Guerin, M. E., Shepard, W., Ugalde, R. A. \& Alzari, P. M. (2004) Crystal structure of glycogen synthase: homologous enzymes catalyze glycogen synthesis and degradation, The EMBO journal. 23, 3196-205.

25. Busi, M. V., Barchiesi, J., Martín, M. \& Gomez-Casati, D. F. (2014) Starch metabolism in green algae, Starch - Stärke. 66, 28-40.

26. Yep, A., Ballicora, M. A. \& Preiss, J. (2004) The active site of the Escherichia coli glycogen synthase is similar to the active site of retaining GT-B glycosyltransferases, Biochemical and biophysical research communications. 316, 960-6.

27. Busi, M. V., Palopoli, N., Valdez, H. A., Fornasari, M. S., Wayllace, N. Z., GomezCasati, D. F., Parisi, G. \& Ugalde, R. A. (2008) Functional and structural characterization of the catalytic domain of the starch synthase III from Arabidopsis thaliana, Proteins. 70, $31-40$.

28. Fontaine, T., D'Hulst, C., Maddelein, M. L., Routier, F., Pepin, T. M., Decq, A., Wieruszeski, J. M., Delrue, B., Van den Koornhuyse, N., Bossu, J. P. \& et al. (1993) Toward an understanding of the biogenesis of the starch granule. Evidence that Chlamydomonas soluble starch synthase II controls the synthesis of intermediate size glucans of amylopectin, The Journal of biological chemistry. 268, 16223-30.

29. Szydlowski, N., Ragel, P., Raynaud, S., Lucas, M. M., Roldan, I., Montero, M., Munoz, F. J., Ovecka, M., Bahaji, A., Planchot, V., Pozueta-Romero, J., D'Hulst, C. \& 
Merida, A. (2009) Starch granule initiation in Arabidopsis requires the presence of either class IV or class III starch synthases, The Plant cell. 21, 2443-57.

30. Deschamps, P., Moreau, H., Worden, A. Z., Dauvillee, D. \& Ball, S. G. (2008) Early gene duplication within chloroplastida and its correspondence with relocation of starch metabolism to chloroplasts, Genetics. 178, 2373-87.

31. Boyer, C. D. \& Preiss, J. (1979) Properties of Citrate-stimulated Starch Synthesis Catalyzed by Starch Synthase I of Developing Maize Kernels, Plant physiology. 64, 103942.

32. Nakamura, Y., Aihara, S., Crofts, N., Sawada, T. \& Fujita, N. (2014) In vitro studies of enzymatic properties of starch synthases and interactions between starch synthase I and starch branching enzymes from rice, Plant science : an international journal of experimental plant biology. 224, 1-8.

33. Jobling, S. (2004) Improving starch for food and industrial applications, Current opinion in plant biology. 7, 210-8.

34. Smith, A. M. (2008) Prospects for increasing starch and sucrose yields for bioethanol production, The Plant journal : for cell and molecular biology. 54, 546-58.

35. Sorokina, O., Corellou, F., Dauvillee, D., Sorokin, A., Goryanin, I., Ball, S., Bouget, F. Y. \& Millar, A. J. (2011) Microarray data can predict diurnal changes of starch content in the picoalga Ostreococcus, BMC systems biology. 5, 36.

36. Hennen-Bierwagen, T. A., Lin, Q., Grimaud, F., Planchot, V., Keeling, P. L., James, M. G. \& Myers, A. M. (2009) Proteins from multiple metabolic pathways associate with starch biosynthetic enzymes in high molecular weight complexes: a model for regulation of carbon allocation in maize amyloplasts, Plant physiology. 149, 1541-59.

37. Edwards, A., Borthakur, A., Bornemann, S., Venail, J., Denyer, K., Waite, D., Fulton, D., Smith, A. \& Martin, C. (1999) Specificity of starch synthase isoforms from potato, European journal of biochemistry / FEBS. 266, 724-36.

38. Senoura, T., Isono, N., Yoshikawa, M., Asao, A., Hamada, S., Watanabe, K., Ito, H. \& Matsui, H. (2004) Characterization of starch synthase I and II expressed in early developing seeds of kidney bean (Phaseolus vulgaris L.), Bioscience, biotechnology, and biochemistry. 68, 1949-60.

39. Damager, I., Denyer, K., Motawia, M. S., Moller, B. L. \& Blennow, A. (2001) The action of starch synthase II on 6"'-alpha-maltotriosyl-maltohexaose comprising the branch point of amylopectin, European journal of biochemistry / FEBS. 268, 4878-84.

40. Furukawa, K., Tagaya, M., Inouye, M., Preiss, J. \& Fukui, T. (1990) Identification of lysine 15 at the active site in Escherichia coli glycogen synthase. Conservation of Lys-XGly-Gly sequence in the bacterial and mammalian enzymes, The Journal of biological chemistry. 265, 2086-90.

41. Furukawa, K., Tagaya, M., Tanizawa, K. \& Fukui, T. (1993) Role of the conserved Lys-X-Gly-Gly sequence at the ADP-glucose-binding site in Escherichia coli glycogen synthase, The Journal of biological chemistry. 268, 23837-42.

42. Schinzel, R. \& Drueckes, P. (1991) The phosphate recognition site of Escherichia coli maltodextrin phosphorylase, FEBS letters. 286, 125-8.

43. Zheng, Y., Anderson, S., Zhang, Y. \& Garavito, R. M. (2011) The structure of sucrose synthase-1 from Arabidopsis thaliana and its functional implications, The Journal of biological chemistry. 286, 36108-18. 


\section{Figure Legends}

Fig. 1. (A) Schematic representation of OsttaSSIII-C. GT1 and GT5 domain are shown. (B) Alignment obtained using the Unipro UGENE v.1.10.4 program (with default parameters) between OsttaSSIII-C, ArathSSIII-CD (Arabidopsis thaliana SSIII catalytic domain), AgrtuGS (Agrobacterium tumefaciens glycogen synthase), EsccoGS (Escherichia coli glycogen synthase), ChlreSSIII1 CD and ChlreSSIII2 CD (Chlamydomonas reinhardtii SSIII 1 and 2, catalytic domains). OsttaSSIII-C amino acids residues R270, K275 and E352 are indicated by open boxes (corresponding to site active amino acids R300, K305 and E377 characterized in EsccoGS $[18,26])$. Other previously characterized catalytic residues are shown in bold. Grey boxes indicate identical residues.

Fig. 2. Homology modelling of OsttaSSIII-C and mutants. (A) Structural model of E. coli GlgA (PDB 2QZS, left) and the proposed model for OsttaSSIII-C (right); (B) Close-up view of catalytic site of OsttaSSIII-C, showing the residues involved in the active site; $(\mathrm{C}$, D, E) Close-up view of catalytic site of OsttaSSIII-C, showing the residues involved in the active site for the mutants R270, K275 and E352 respectively.

Fig. 3. (A) SDS-PAGE of recombinant enzymes. Lane 1: Molecular weight standard, lane 2, OsttaSSIII-C; lane 3, OsttaSSIII-C R270A; lane 4, OsttaSSIII-C K275A; and lane 5, OsttaSSIII-C E352A. Numerals indicate the molecular masses (MM) of the standards (Prestained SDS-PAGE Standards, Low Range, BioRad).

Fig. 4. ADPGlc (A), glycogen (B) and amylopectin (C) saturation plots for OsttaSSIII-C determined in the presence of $2 \mathrm{mg} / \mathrm{ml}$ glycogen (A) or $500 \mu \mathrm{M}$ ADPGlc (B and C).

Fig. 5. (A) Effect of $\mathrm{pH}$ on the activity of OsttaSSIII-C. $50 \mathrm{mM}$ Bicine buffer was used for pH 6.0-10.0. Assays were run with $2 \mathrm{mg} / \mathrm{ml}$ glycogen and $1 \mathrm{mM}$ ADPGlc. Data are relative values compared with activity at $\mathrm{pH}$ 8. (B) Effect of temperature on the activity of OsttaSSIII-C. Assays were run with $2 \mathrm{mg} / \mathrm{ml}$ glycogen and $1 \mathrm{mM}$ ADPGlc, in $50 \mathrm{mM}$ Bicine buffer, with reaction temperatures of $20,25,30,35,40$ and $45{ }^{\circ} \mathrm{C}$. Data are relative values compared with activity at $35^{\circ} \mathrm{C}$. 
Fig. 6. Far-UV Circular Dichroism Spectra of recombinant and mutated OsttaSSIII-C isoforms. Wild type protein is represented by dotted lines. OsttaSSIII-C R270A, K275A and E352A are represented by dashed line, solid line and bold dotted line, respectively. Circular Dichroism spectra were obtained in $20 \mathrm{mM}$ Tris- $\mathrm{HCl}$ buffer, $\mathrm{pH}$ : 7.5. 